\title{
Raimund Ottow
}

\section{Der missverstandene Richard Hooker}

\author{
Kritik an: Christof Breitsameter: "Richard Hooker. Zur Frage nach den normativen \\ Voraussetzungen des modernen Rechtsstaates " in: Zeitschrift für Politik, 53. Jg. \\ 2006 Heft 1, S. 74-90.
}

Es ist heutzutage nicht sehr verbreitet, in akademischen Zeitschriften Kontroversen zu führen, aber der genannte Aufsatz ist so verkehrt, dass ich auf eine Kritik nicht verzichten möchte. Da ich mich für Richard Hooker und seine englischen Zeitgenossen interessiere, habe ich den Text gelesen, der aber mit Hooker so gut wie nichts zu tun hat. Stattdessen wird ihm (mit der lahmen Entschuldigung, Hooker sei terminologisch nicht sehr klar, er schwanke zwischen Mittelalter und Moderne, u.ä.) eine moderne Position angedichtet und aufgepropft, die aus Hobbes, Pufendorf, Kant, James Buchanan, Niklas Luhmann u.a. Autoren gewonnen ist, die an sich in diesem oder jenem Aspekt interessant sein mag, aber, wie gesagt, so gut wie nichts mit Hooker zu tun hat. Bezeichnend ist auch, dass der Verfasser die einschlägige Literatur ignoriert. ${ }^{1}$ So kann man Geschichte politischen Denkens als wissenschaftliche Disziplin nicht betreiben.

1 Donald W.Hanson, From Kingdom to Commonwealth. The development of Civic Consciousness in English Political Thought, Cambridge (Mass.) 1970, S.265ff.; W.D.J. Cargill Thompson, »The Philosopher of the >Politic Society : Richard Hooker as a Political Thinker « zuerst 1972, dann in: ders., Studies in the Reformation: Luther to Hooker, hg.v. C.W.Dugmore, London 1980; Robert Eccleshall, Order and Reason in Politics. Theories of Absolute and Limited Monarchy in Early Modern England, Oxford 1978, Kap.5; Arthur B. Ferguson, Clio Unbound. Perception of the social and cultural past in Renaissance England, Durham 1979, S.207-21, 249ff.; Peter Lake, Anglicans and Puritans? Presbyterianism and English Conformist Thought from Whitgift to Hooker, London 1988, Kap.4; Arthur Stephen McGrade, Of the Laws of Ecclesiastical Polity, Cambridge 1989; ders. (Hg.), Richard Hooker and the Construction of Christian Community, Tempe (Ariz.) 1997; W.J. Torrance Kirby, Richard Hooker's Doctrine of the Royal Supremacy, Leiden u.a.O. 1990; Peter White, Predestination, policy and polemic. Conflict and consensus in the English Church from the Reformation to the Civil War, Cambridge 1992, Kap.7; Richard Helgerson, Forms of Nationhood. The Elizabethan Writing of England, Chicago/London 1992, S.269-83; Richard Tuck, Philosophy and Government, 1572-1651, Cambridge 1993, S.146ff.; Diarmaid MacCulloch, Die zweite Phase der englischen Reformation (1547-1603), und die Geburt der anglikanischen Via Media, Münster 1998, S.102-5; Ders., »Richard Hooker's Reputation « in: The English Historical Review, 117, 2002, S.773-812; Beiträge von Debora Shuger und William J.Bouwsma, in: Claire McEachern / Debora Shuger (Hg.) Religion and Culture in Renaissance England, Cambridge 1997; Alan Cromartie, »Theology and Politics in Richard Hooker's Thought « in: History of Political Thought, 21, 2000, S.41-66. 
"Der Frage, wie viel Autonomie Hooker der Religion, konkreter: der sichtbaren Kirche zugesteht, damit sich die Spiritualität der unsichtbaren Kirche entfalten kann, kann bier nicht weiter nachgegangen werden. Sie wird auch von Hooker nicht gelöst. Sein Grundanliegen ist philosophischer Natur... «, sagt Breitsameter (S.89). Dabei heisst Hookers Werk: >Of the Laws of Ecclesiastical Polity<. Wenn dieses Werk nicht vom Verhältnis Kirche - Staat, oder Kirche - Politik handelt: wovon denn sonst? Und natürlich bezieht sich auch der Verfasser auf diese Frage.

Sehen wir uns eine Stelle an: "Die sichtbare Kirche", schreibt Breitsameter, »ist, wie der Staat auch, Teil der Gesellschaft. Ein und dieselbe Person kann Mitglied der Kirche und Mitglied des Gemeinwesens sein. Es gibt nur eine gesellschaftliche Ordnung. Diese Ordnung wird durch Normen hergestellt, die der Vernunft entspringt. Das schließt natürlich nicht aus, dass Kirche und Gemeinwesen für unterschiedliche Lebensbereiche unterschiedliche Normen hervorbringen. (. .) Religion und Politik, so könnte man auch sagen, erweisen sich in dieser frübmodernen Konzeption als autonome Teilbereiche sozialer Ordnung. "Woher nimmt der Verfasser hier die Unterscheidung von Staat und Gesellschaft? Was ist Hookers >Polity`, oder, der Terminus, den andere Zeitgenossen bevorzugten: das >Commonwealth (der Verfasser spricht dann selbst vom >Gemeinwesen $:$ ist das Staat, oder ist es Gesellschaft?) anderes als eben jene vormoderne Konzeption, die diese Unterscheidung grundlegend verneint? Meint das der Verfasser mit dem Satz: »Es gibt nur eine gesellschaftliche Ordnung «? - die demnach Staat (oder Politik) und Kirche umgreift. Dann aber folgt im Text die Behauptung, dass »Kirche und Gemeinwesen für unterschiedliche Lebensbereiche unterschiedliche Normen hervorbringen « können. Ist >Gemeinwesen hier Staat, oder Gesellschaft, oder beides? Und wenn das > Gemeinwesen< beides ist, warum ist dann die Kirche auf einmal nicht mehr Teil der Gesellschaft, sondern ein >unterschiedlicher Lebensbereichく, oder, noch schärfer formuliert, ein sautonomer Teilbereich sozialer Ordnunge? Warum handelt Hooker von einer secclesiastical Polity<? Ein heilloses Durcheinander von Begriffen, in das nur Ordnung zu bringen ist, wenn wir uns die Situation klar machen.

Seitdem sich Henry VIII zum >Head of the Church of England hatte erklären lassen (>under Christ ‘ oder >on earth König als Haupt der Kirche den Zeitgenossen als Blasphemie erschienen wäre) war klar, dass die Kirche nicht ein sunterschiedlicher Lebensbereich oder gar sautonomer Teilbereich sozialer Ordnung ‘ war, sondern der Kontrolle durch die Krone unterlag. Elizabeth als Frau war im auch religiös abgestützten patriarchalen Verständnis der Zeit schwerer als >Head of the Church legitimierbar; trotzdem hielt man an dem Modell der Staatskirche fest, wobei das unleidliche einer Frau als Kirchenhaupt dadurch abgemildert wurde, dass sie sich nurmehr als >Governor der Kirche bezeichnen ließ. ${ }^{2}$ Und diese Konstruktion will Hooker gegen die Puritaner verteidigen und legitimieren. Das achte und letzte Buch von Hookers >Laws< dient speziell dem Zweck der Legitimierung des Kirchensupremats der Krone. Der König/die Königin ist Haupt sowohl der weltlichen/politischen Ordnung als auch gleichzeitig Haupt

2 Siehe meinen Text in: Zeitschrift für Politik, 52.Jg., 2005, Heft 2, S.190-207. 
der Kirche: dadurch erst wird die Ordnung der englischen >Polity zu einer >ecclesiastical Polity`, deren beide Ordnungsstränge, der weltlich-poltische und der kirchliche, in der Krone zusammenlaufen. Und das Beweisziel Hookers ist, dass die Puritaner dieser Staatskirche unter der Krone aus guten Gründen gehorchen müssen. Daher ist die Behauptung des Verfassers absurd, bei Hooker könne man von einem »Modell eines säkularen Staates « sprechen (S.88). Man stelle sich vor, Horst Köhler wäre >Haupt der Kirche von Deutschland , der die Bischöfe ernennt, die Synoden einberuft, den Beschlüssen der Synoden sein Placet gibt, usw.! Denn das ist es, was Elizabeth getan hat und was Hooker legitimieren will.

Da der Verfasser offensichtlich keine Ahnung hat, wie die englische Konstellation war, kommt er zu Thesen über Religionsfreiheit, Gewissensfreiheit und Meinungsfreiheit als Menschenrechte bei Hooker, die in Hookers Text kein Fundament haben. Hooker soll sich auf das Vorbild der Niederlande bezogen haben, die tatsächlich keine Staatskirche im strengen Sinne, sondern nur eine privilegierte sogenannte ১Öffentlichkeitskirche< (die niederländischen Reformierten) und daneben begrenzte Religionsfreiheit hatten. Aber das ist nicht Hookers Modell: seine secclesiastical Polity< meint nicht eine Gesellschaft mit mehreren Bekenntnissen und Kirchen, denen gegenüber sich der Staat relativ neutral verhält, sondern meint eine Personalidentität von Staatsvolk und Kirchenvolk, Deckungsgleichheit von >Polity< und Kirche, die als recclesiastical Polity< beide von der Krone regiert werden.

Dem Verfasser zufolge "vertritt Hooker die Meinung, dass der Mensch in religiösen wie in politischen Fragen selbständig und eigenverantwortlich entscheiden kann und dass beide Sphären klar getrennt werden müssen. Jeder Mensch kann für sich Gewissensfreibeit in Anspruch nehmen und hat im Gegenzug die abweichenden Überzengungen anderer Menschen zu respektieren." (S.81) Offensichtlich ist dem Verfasser nicht bekannt, dass sogenannte Separatisten (’Brownists<, >Barrowists<, John Greenwood u.a.) in England verfolgt wurden, z.T. im Gefängnis landeten, einige auch hingerichtet wurden. Und es gibt keinerlei Anhaltspunkte dafür, dass Hooker (abgesehen davon, dass er vielleicht für moderate Methoden der Verfolgung plädiert hat) diese Verfolgung aus prinzipiellen, theoretischen Gründen abgelehnt oder kritisiert hätte. Wie denn auch, wenn das Grundmodell die Einheit von Staatsvolk und Kirchenvolk ist? Jeder Separatismus von der englischen Staatskirche mußte ihm notwendig als schismatisch erscheinen.

Richtig ist, dass Hooker gegen die Puritaner die Vernunft stark gemacht hat, aber nicht die individuelle Vernunft, die sich alles mögliche einbilden mag und in Schismen führt, sondern die kollektive Vernunft der >ecclesiastical Polity<. Der Streitpunkt mit den Puritanern (über den man bei Breitsameter wenig erfährt) lag darin, dass die Puritaner die englische Kirche in einer Reihe von Aspekten: Zeremonien, Kirchenorganisation u.a., als nur halb reformiert und noch halb spapistisch kritisierten, dass also die gegebene Form und Praxis der englischen Kirche im Widerspruch zum Wort Gottes stehe. Darüber wurde spätestens seit 1572, dem Jahr der Publikation der puritanischen >Admonitions to the Parliament $<$, in England intensiv diskutiert. ${ }^{3}$ Hookers >Laws of Ecclesiastical Polity< sind ein später Beitrag zu dieser Debatte. Und seine These lautet, dass die >Heiligen Schriften` (es gab Diskussionen 
darüber, welche Texte genau dazu gehören; aber davon abgesehen) keine hinreichende Grundlage dafür hergeben, wie die Kirche in ihrer äußerlichen Gestalt - Organisation, Zeremonien usw. - zu gestalten sei. Vielmehr müsse davon ausgegangen werden, dass Gott den Menschen (aber nicht den Individuen, sondern der jeweiligen recclesiastical Polity`) die Freiheit zugeschrieben hat, diese Fragen den Zeitumständen gemäß nach ihrer menschlichen Vernunft zu entscheiden.

In diesen Zusammenhang gehört die von Breitsameter ordentlich verwirrte Unterscheidung von >things necessary von `things indifferent<, die nicht sonderlich originell bei Hooker ist, sondern, auch unter der Form der Rede von den >Adiaphora<, zum akzeptierten Begriffsarsenal des Protestantismus gehörte. Man unterschied damit, was zum notwendigen, unerlässlichen und unveränderlichen Kernbestand des 'wahren Glaubens` gehört, der gewahrt werden muß, wenn die Gläubigen auf Erlösung sollen hoffen können, von den Elementen der äußerlichen Kirchenordnung, die veränderbar sind. Die Puritaner bestritten diese Unterscheidung, oder sie bestritten, dass die Establishment-Autoren die Grenze richtig zogen, aber diese ganze Debatte hat jedenfalls nichts mit individueller Religions-, Gewissens- und Meinungsfreiheit zu tun, denn sowohl für die Establishment-Autoren, wie Hooker, als auch für die Puritaner war klar, dass sich die einzelnen Gläubigen der Entscheidung der Kirche beugen müssen. »I would therefore know «, fragt Hooker an einer Stelle seines Vorwortes die Puritaner, »whether for the ending of these irksome strifes wherein you and your followers do stand thus formally devided against the authorized guides of this Church, and the rest of the people subject unto their charge, whether I say ye be content to referre your cause to any other higher judgement then [i.e.: than] your owne, or else intend to persist and proceed as ye have begun, til your selves can be perswaded to condemne your selves. « Er verlangt also von den Puritanern, eine Entscheidung der Kirche zu akzeptieren, auch wenn und obwohl sie nicht überzeugt sind. Die Einheit von Staatsvolk und Kirchenvolk, die Einheit der >ecclesiastical Polity< aber stand für keine der beiden Seiten in Frage.

Breitsameter will unter den >things necessary « Grundwabrbeiten [verstehen], die menschlicher Beschlussfassung entzogen sind.« (S.82) Warum? Weil es Grundwahrheiten des Glaubens sind, die niemand in Frage stellen darf, weil er sonst Gefahr läuft, soteriologisch betrachtet, sein Seelenheil zu verlieren, und äußerlich betrachtet, mit Geldstrafen belegt, inhaftiert oder, schlimmsten Falls, als Häretiker hingerichtet zu werden (was einigen Wiedertäufern zur Zeit von Königin Elizabeth passierte). Hooker: »This not to believe is eternal death and damnation, or, This everie soule that will live must duly observe, of which sort the articles of Christian fayth, and the sacramentes of the Church of Christ are, all such thinges if scripture did not

3 Greifbar in Walter H.Frere / C.E.Douglas (eds.): Puritan Manifestoes. A Study of the Origin of the Puritan Revolt, with a Reprint of the Admonition to the Parliament and kindred Documents, 1572, (zuerst 1907) Reprint, London 1954; siehe sodann die Kontroverse zwischen Thomas Cartwright und John Whitgift, greifbar in: The Works of John Whitgift, hg.v. John Ayre, The Parker Society, 3 Bde., Cambridge 1851-53.

4 The Folger Library Edition of the Works of Richard Hooker, Cambridge (Mass.)/London, Bd.1, S.29f. 
comprehende, the Church of God should not be able to measure out the length and the breadth of that waye wherein for ever she is to walke, Heretiques and Schismatiques never ceasing some to abridge, some to enlarge, all to pervert and obscure the same. $\ll^{5}$ Auf unerklärliche Weise jedoch verwandeln sich diese >Grundwahrheiten bei Breitsameter in "unverfügbare Rechte «, und zwar solche, »die sicherstellen, dass sich auch in majorisierten Fragen Minderbeiten zu Wort melden können, so dass künftig andere Mebrheitsverbältnisse möglich sind.«(S.82) Das hat, sosehr dies ein sympathischer Gedanke ist, nichts mit der Unterscheidung zwischen >things necessary und sthings indifferent bei Hooker und seinen Zeitgenossen zu tun. Das Gegenteil trifft zu: >things necessary< sind Kernbestandteile des Glaubens, über die es in einer wohlgeordneten secclesiastical Polity< keine Meinungsverschiedenheiten und keine Mehrheits- und Minderheitenmeinungen geben kann/darf. Allenfalls bei things indifferent sind Meinungsverschiedenheiten möglich. Hooker schreibt, die zitierte Stelle fortsetzend: »But as for those thinges that are accessorie hereunto... in such thinges because discretion may teach the Church what is convenient, we holde not the Church further tyed herein unto scripture [wie die Puritaner verlangten], then [i.e.: than] that against scripture nothing be admitted in the Church... Is it then in that wee accompt Ceremonyes (...) is it an oversight, that wee recken these thinges and matters of governement in the number of thinges accessorie, not things necessarie... ? $\ll^{6}$

Dabei ist zu beachten, dass es die Kirche ist, die über sthings indifferent/accessorie< entscheidet, nicht etwa Individuen oder Gruppen, die sich der Entscheidung der Kirche vielmehr zu beugen haben. Und warum? Weil, und hier liegt allenfalls die Originalität Hookers, die auch für die Geschichte des politischen Denkens von Interesse ist, er die Entscheidung der Kirche als eine auf Konsensprozessen beruhende (eventuell Mehrheits-) Entscheidung der ganzen secclesiastical Polity« rekonstruiert. Denn England hat, im Gegensatz zu anderen Ländern, eine konsensual angelegte Regimeform, zum einen dadurch, dass nach Hooker der Erbmonarchie eine ursprüngliche Entscheidung der Gemeinschaft für diese Regimeform und diesen oder jenen Ursprungsdynasten notwendig zugrunde liegen muss, die auch die Nachkommen bindet, zum anderen dadurch, dass die Erbmonarchie durch das Parlament, das den >Körper des Reiches repräsentiert, konsensual limitiert wird. Und abgesehen davon, dass die Zuschreibung, Haupt der Kirche zu sein, von Henry VIII eventuell auch auf seine Nachfolger auf dem Thron überging, hatte das Parlament Elizabeth zu Beginn ihrer Regierungszeit als >Governor< der Kirche eingesetzt oder bestätigt und sie gesetzlich mit den notwendigen Vollmachten ausgestattet, die Kirche tatsächlich zu regieren. Und das Parlament hatte zu dieser Zeit das >Common Prayer Book und 1563 die >Thirty-Nine Articles zusammen mit weiterem Material als Grundlagen der englischen Kirche und Kirchenordnung gebilligt. Zwar war nun staatsrechtlich nicht ganz klar, ob die Kirchenführer im Oberhaus als Vertreter des Klerus oder als >ecclesiastical barons« saßen, die durch ihre Ernennung zu Bischöfen

5 Ibid., S.211.

6 Ibid., S.211f.

ZfP 54. Jg. 4/2007 
Landeigentum der Krone im Namen und zum Nutzen der Kirche verwalteten, aber unabhängig davon hatten auch die beiden Synoden von Canterbury und York, in denen der Klerus in personam oder durch Vertreter repräsentiert war, die englische Kirchenordnung gebilligt, so dass niemand im Reich, ob Kleriker oder Laie, ob Puritaner oder nicht, sagen konnte, er sei nicht (nach zeitgenössischem Verständnis, also weit entfernt von allgemeinen, gleichen und geheimen Wahlen) direkt oder indirekt bei der Verabschiedung der Kirchenordnung repräsentiert gewesen. Wenn sich also die Puritaner auch nach 20-jähriger Debatte nicht der Entscheidung des ganzen Reiches beugen wollten, wollte ihnen Hooker klar machen, dass die unanfechtbar legitimierte Konsensentscheidung des Reiches in seinen beiden Teilen: Laienschaft und Klerus, die Entscheidung also der secclesiastical Polity< in ihrer Gesamtheit auch sie band - ob sie nun überzeugt waren, oder nicht. Dass Leute, die um ihr Seelenheil fürchten, wenn sie sich einer gottwidrigen Kirche unterwerfen, sich durch konsensual legitimierte Verfahren nicht unbedingt beeindrucken lassen, hat die weitere Geschichte deutlich gemacht.

Ein interessanter Punkt des Textes von Breitsameter soll abschließend angesprochen werden. Wenn die Entscheidung der secclesiastical Polity< in sthings indifferent nicht notwendig, sondern durch Klugheitsgesichtspunkte und dergleichen bestimmt ist, dann liegt tatsächlich auf der Hand, dass durch veränderte Zeitumstände, durch neue Einsichten und anderes eine Änderung der Kirchenordnung in things indifferent angezeigt sein kann, und also muss es Wege, Kanäle geben, durch die diskursiv solche Änderungen initiiert werden können. Hat also Breitsameter doch Recht mit seiner These von Hooker als Vertreter von >Meinungsfreiheit<? Ja und Nein. Nicht-christliche Religionen, christliche Häresien jenseits des Kerns des christlichen Glaubens, schismatische und separatistische Bestrebungen sind seinem Modell der Einheit der secclesiastical Polity zufolge ausgeschlossen. Breitsameters Satz: »Nach Hooker kann jeder Gläubige irgendeines Bekenntnisses im Bereich der ddaily things politisch mit bestimmen « (S.87), ist falsch. Ebenso schloß Hooker eine Fundamentalkritik der Kirche auf puritanischen Linien aus, die die von der recclesiastical Polity legitimierte und getragene Kirche als spapistisch anti-christlich usw. denunziert und praktisch die Unterwerfung unter die secclesiastical Polity< verweigert. Auf der anderen Seite, und vielleicht gerade weil er selbst so bestürzt war über den blinden Dogmatismus der Puritaner, die jedes Komma von Calvin und Theodor Beza nachbeteten, plädierte er dafür, jedenfalls die theologischen Fakultäten und genereller die gelehrte Diskussion der Theologen diskursiv zu öffnen, so dass ein offener und innerhalb gewisser Grenzen pluraler Umgang mit dem theologischen Material möglich wird. Im übrigen würde er die Kritiker der Kirche auf die normalen Wege der Entscheidungsfindung der secclesiastical Polity< verweisen: das Parlament und die >Convocations< des Klerus. Elizabeth hat diversen ihrer Parlamente die Befassung mit Kirchenfragen untersagt, und es kann sein, dass Hooker das kritisch gesehen hat und insofern als 'parlamentarischer Kritiker des Kirchensupremats der Krone gelten könnte, aber das ist, wenn überhaupt, schwer beweisbar. 